\title{
Evaluación de la calidad de los registros empleando la auditoría odontológica en una clínica dental docente.
}

Quality evaluation of the records using dental audit in a teaching dental clinic

\author{
Jorge A Manrique Guzmán ${ }^{1, a}$, Jorge E Manrique Chávez ${ }^{2, a}$, Beatriz Chávez Reáteguỉ ${ }^{3, b}$, Carolina B Manrique \\ Chávez ${ }^{4, c}$.
}

\section{RESUMEN}

Objetivo: Evaluar la calidad de registro o llenado de historias clínicas de pacientes nuevos atendidos en una Clínica Dental Docente durante el período de enero a julio de 2012 empleando la auditoría odontológica como herramienta sistematizada. Material y métodos: Se tomaron como muestra 140 historias clínicas de pacientes nuevos de las cuales se evaluó la frecuencia y porcentaje de registro de los diferentes aspectos que componen la historia clínica. Resultados: Se encontró que el 78\% de las historias no tenían completo el registro en los diferentes aspectos evaluados, encontrando que de los diecisiete aspectos evaluados, nueve registran entre $11 \%$ y $29 \%$, quedando entre un $89 \%$ y un $71 \%$ de historias que no registran estos aspectos. Se identificaron tres aspectos que se registran entre $84 \%$ y $86 \%$ (número de historia, cuestionario de salud y examen estomatológico), cuatro aspectos registran entre $94 \%$ y $98 \%$ (fecha de ingreso, asignación de operador, odontograma y filiación) y sólo un aspecto se registra al 100\% (evolución del tratamiento) del total de historias clínicas auditadas. Conclusiones: Más del $45 \%$ de las historias clínicas auditadas en la presente investigación tenían deficiencias en el registro o llenado de los datos que la historia clínica requiere como información de documento médico-legal.

\section{Palabras clave: AUDITORÍA MÉDICA, AUDITORÍA DENTAL, AUDITORÍA CLÍNICA, HISTORIA CLÍNICA.}

\section{SUMMARY}

Objective: To assess the quality of registration or filling histories of new patients treated at a Teaching Dental Clinic during the period January to July 2012 using the Dental Audit as a tool systematized. Methods: Was sampled 140 medical records of new patients which assessed the frequency and percentage of recording various aspects that make up the medical record. Results: We found that $78 \%$ of the medical records do not have full registration in the different aspects evaluated, finding that of the seventeen aspects evaluated, nine recorded between $11 \%$ and $29 \%$, remaining between $89 \%$ and $71 \%$ of medical records that not record these aspects. We identified three aspects that are recorded between $84 \%$ and $86 \%$ (medical record number, health questionnaire

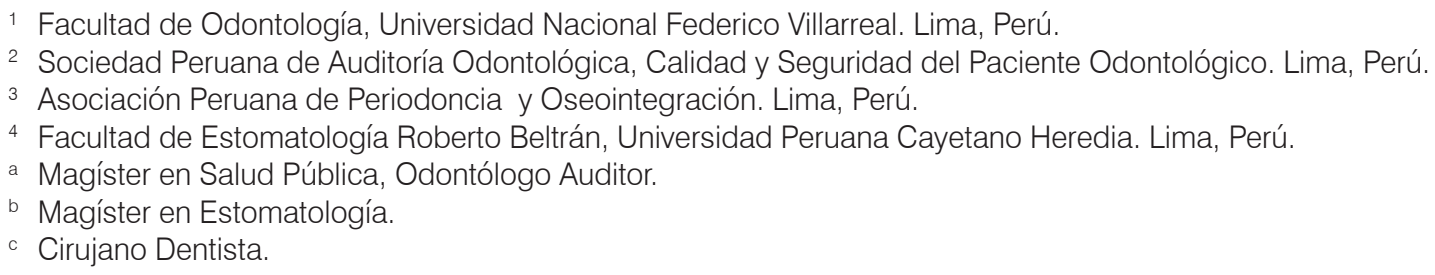


and dental examination), four points recorded between $94 \%$ and $98 \%$ (date of entry, operator assignment, odontogram and filiation) and only one side is recorded 100\% (treatment outcome) of the total audited medical records. Conclusions: Over 45\% of medical records audited in this investigation are impaired in the registry or data filling medical history required as medico-legal document.

\section{Keywords: MEDICAL AUDIT, DENTAL AUDIT, CLINICAL AUDIT, MEDICAL RECORD.}

\section{INTRODUCCIÓN}

La auditoría en servicios en salud no se aparta de las auditorías que se realizan en otros campos del quehacer humano en cuanto a proceso, objetivos, evaluación e informe, constituyéndose en un instrumento de perfeccionamiento y educación continua que permite tomar resguardos en aspectos técnicos, administrativos, éticos y médico-legales en búsqueda de la calidad y/o excelencia en los servicios (1).

La auditoria en salud, al trabajar sobre la base de registros de lo efectuado, no solo evalúa la calidad de estos, sino también valora la calidad de la atención misma, demostrando que hay una relación directa entre la calidad de los registros y la de la atención brindada. La auditoría en los servicios de salud se fue derivando en una serie de ramas enfocadas en determinadas áreas de la salud, en la cual destacan la auditoría médica relacionada al profesional exclusivamente médico y la auditoría clínica relacionada a los profesionales de salud en general (no sólo médicos), de esta última nace la auditoría odontológica enfocada al área del componente bucal de la salud (2).

La auditoría médica ha sido utilizada durante mucho tiempo y una de sus definiciones mas ampliamente aceptadas y reconocidas es la descrita en 1989 por el National Health Service: "Es el análisis crítico sistemático de la calidad de la atención médica, incluyendo procedimientos diagnósticos y decisiones terapéuticas, el uso de recursos y los resultados de los mismos que repercutan en los desenlaces clínicos y en la calidad de vida del paciente" (Secretaries of State for Health, Wales, Northern Ireland and Scotland). La auditoría médica se puede llevar a cabo de diversas formas y a diferentes niveles durante la atención del paciente, por ejemplo, en la revisión de historias clínicas para determinar el registro o calidad de registro de las mismas, en el análisis de reporte de caso de muertes y complicaciones para el caso médico o accidentes y complicaciones para el caso odontológico y su manejo ambulatorio u hospitalario, entre otros (3).

La auditoría clínica busca una revisión de la actividad de la salud en todos los aspectos de cuidado y atención clínica realizado por profesionales de salud, tanto médico como no médico y se aprovecha su uso como un instrumento de garantía de calidad de los servicios de salud que permite la evaluación de sus objetivos al tiempo que se convierte en un componente de la intervención para mejorarlos (4-6). La auditoría enfocada al campo de la salud, se desarrolla como un proceso de mejora continua que determina la calidad de atención en comparación con estándares e inicia acciones de mejora cuando éstos no son alcanzados $(7,8)$.

La auditoría odontológica es una disciplina científicaque sehadesarrolladodemanera considerable en el transcurso de las últimas décadas, producto de cambios en el sector salud y a raíz de la incorporación de los conceptos básicos del proceso administrativo al ejercicio profesional. La auditoría en los servicios de salud es clasificada en función al objeto a auditar, así tenemos auditoría de caso que se desarrollada en las diferentes especialidades médicas siguiendo una serie de procedimientos sistemáticamente diseñados para la auditoría frente a un caso clínico que requiera ser sujeto a auditoría determinar su conformidad, no conformidad u observaciones durante el manejo, la auditoría de fuentes secundarias que tiene como objeto de análisis principalmente a la historia clínica pero además puede expandirse a todos aquellos documentos que en conjunto pueden dar origen al expediente clínico como son las fichas de evolución, hojas de interconsultas, exámenes de laboratorio, etc. Otro enfoque donde la auditoría se desarrolla es la llamada auditoría basada en la evidencia que permite la evaluación de una atención o grupo de atenciones sobre el paciente en un periodo de tiempo con una orientación basada en la evidencia científica que permita verter un informe que sustente su intervención. 
Por otro lado, en función a la temporalidad, las auditorías se clasifican en auditorías retrospectivas y auditorías prospectivas o concurrentes. Para el caso de las auditorías de las historias clínicas, se han considerado dos momentos, un primer momento, relacionado con el registro de las historias, donde no se discute la calidad de información sino la existencia de la misma, centrándose en este registro, mientras que el segundo momento se centra en la calidad de la información registrada (9).

La historia clínica es uno de los elementos fundamentales para el trabajo médico, y es la pieza clave en toda auditoría de servicios de salud. Es importante por cuanto contiene datos acerca de lo que se ha encontrado en un paciente, lo que se ha pensado y lo que se ha hecho por su atención $(10,11)$. Es el instrumento más útil para valorar la calidad de atención brindada a los pacientes pues es la biografía médica/odontológica de ellos $(12,13)$, permite una recolección ordenada de datos de identidad, síntomas, signos y otros elementos que permiten al profesional de la salud plantear un diagnóstico clínico, sindrómico o nosológico. La historia clínica es un documento con valor académico, asistencial y legal, que consigna una serie de datos clínicos referidos a una persona, con el propósito de determinar un diagnóstico correcto y, en consecuencia, el plan de tratamiento conveniente que pueda concluir en la intervención, medicación o tratamiento apropiado, pertinente y oportuno de la persona afectada (8).

En el campo odontológico, la historia clínica debe considerarse el documento básico en todas las etapas del modelo de atención y ser apreciada como una guía metodológica para la identificación integral de los problemas del componente bucal de la salud de cada persona. Se emplea para el planeamiento, ejecución y control de las acciones destinadas a la prevención, recuperación, y rehabilitación (14).

La presente investigación se centra en la auditoría odontológica como proceso sistemático para la evaluación de la calidad de registros odontológicos (historias clínicas) de pacientes nuevos atendidos entre enero-julio de 2012 en una clínica dental docente cuya misión es formar profesionales odontólogos así como brindar servicio de asistencia odontológica, por lo que se encuentra comprometida tanto en la calidad de la educación como con la calidad de la atención sanitaria que brinda.

\section{MATERIAL Y MÉTODOS}

Se empleó instrumento diseñado especialmente diseñado para la investigación a cada historia clínica de pacientes nuevos atendidos entre Enero-Julio de 2012 en la Clínica Dental Docente como parte del proceso de Auditoría Odontológica, excluyéndose historias clínicas de pacientes continuadores atendidos en la clínica dental fuera del periodo de estudio, así como, historias clínicas de pacientes transferidos de otros servicios. Se consideró el máximo tamaño muestral posible de historias clínicas, al no tenerse referencias en la literatura sobre estudios similares de auditoría odontológica de registro de historias clínicas en una clínica docente, se aplicó la ecuación: $N=Z \alpha 2(p * q)$ $/ \mathrm{E}$, donde $\mathrm{Z} \alpha$ es el coeficiente de confiabilidad al $95 \%$ $(1,96)$, p registros correctos es $0,1(10 \%)$ y q es $(1-p)$, E es el error de precisión (considerado 0,05 ), total 139.

Se tomaron como muestra 140 historias clínicas de pacientes nuevos, en las cuales se evaluó el porcentaje de llenado de los diferentes aspectos o ítems que la componen $\left(\mathrm{N}^{\mathrm{o}}\right.$ de historia clínica, fecha de ingreso, filiación, riesgo, asignación, motivo de consulta, cuestionario de salud, examen general, examen estomatológico, odontograma, plan de trabajo para el diagnostico, examen radiológico, presentación de caso, diagnostico, plan de tratamiento, programación y evolución). La relación de pacientes nuevos atendidos se obtuvo del sistema de registro de pacientes del cual tomó el número de muestra previamente establecido aplicando el muestreo aleatorio simple con reemplazo del inmediato inferior del listado. Las historias clínicas fueron solicitadas del archivo central de la Clínica Dental Docente. Los datos se almacenaron en una base de datos generada en el software estadístico IBM SPSS Statistics 20.0 (IBM).

\section{RESULTADOS}

Se encontró que el 78\% de las historias no tienen completo el registro en los diferentes aspectos evaluados (Gráfico 1), de los cuales solo nueve de los diecisiete aspectos (riesgo, motivo de consulta, examen general, plan de trabajo para el diagnóstico, examen radiográfico, presentación de caso, diagnóstico, plan de tratamiento y programación) registran entre $11 \%$ y $29 \%$, quedando entre un $89 \%$ y $71 \%$ de historias clínicas que no evidencian registrados de estos aspectos. Por otro lado, se evidenció que tres 


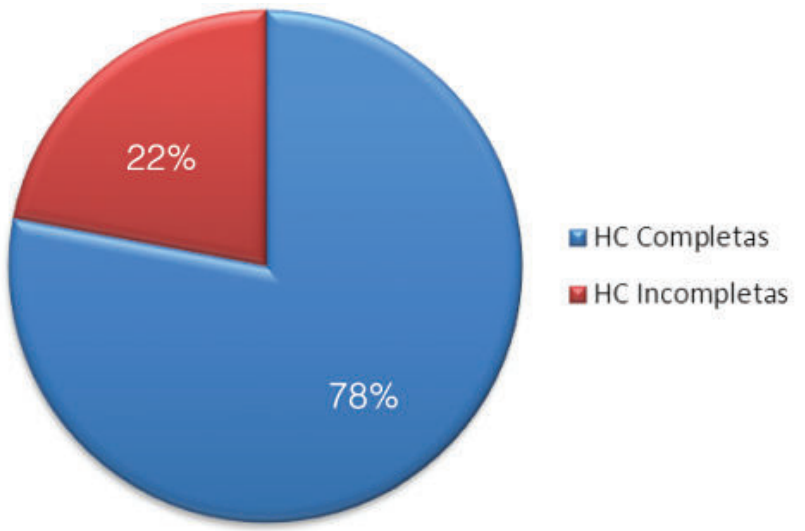

Gráfico 1. Distribución de Historias Clínicas auditadas. El 78\% de las historias con registros incompletos.

aspectos (número de historia, cuestionario de salud y examen estomatológico) se registran entre $84 \%$ y $86 \%$, dejando como resultado que entre $14 \%$ y $16 \%$ de historias clínicas no registran estos aspectos. También se encontró que cuatro aspectos (fecha de ingreso, asignación de operador, odontograma y filiación) registran entre $94 \%$ y $98 \%$, dejando entre un $2 \%$ y $6 \%$ de historias clínicas sin estos ítems. Finalmente, se encontró que sólo un aspecto (evolución del tratamiento) se encuentra registrada en el $100 \%$ de historias clínicas evaluadas durante la auditoría odontológica (Tabla 1). Se observa que hay una relación inversamente proporcional entre la presencia y ausencia de registros en las historias clínicas caracterizados por una amplia brecha entre los registros con altos porcentajes de llenado completo o presente versus los registros con bajos porcentajes de llenado incompleto o ausente (Gráficos 2 y 3 ).

\section{DISCUSIÓN}

La auditoría enfocada al campo de la salud, se desarrolla como un proceso de mejora continua que determina la calidad de atención en comparación con estándares e inicia acciones de mejora cuando éstos no son alcanzados. La auditoria de registros en salud, no solo evalúa la calidad de dichos registros sino que a través de ello también valora la calidad de la atención misma, demostrando que hay una relación directa entre la calidad de los registros y la de la atención brindada.

Tabla 1. Aspectos auditados de las historias clínicas

\begin{tabular}{ccccc}
\hline ASPECTOS & PRESENCIA DE REGISTRO & \multicolumn{2}{c}{ AUSENCIA DE REGISTRO } \\
\hline Programación & $\mathbf{N}^{\mathbf{0}}$ & $\mathbf{\%}$ & $\mathbf{N}^{\mathbf{0}}$ & $\mathbf{\%}$ \\
Examen general & 15 & $10,7 \%$ & 125 & $89,3 \%$ \\
Plan de tratamiento & 20 & $14,3 \%$ & 120 & $85,7 \%$ \\
Riesgo & 20 & $14,3 \%$ & 120 & $85,7 \%$ \\
Plan de trabajo para diagnóstico & 20 & $14,3 \%$ & 120 & $85,7 \%$ \\
Examen radiográfico & 25 & $17,9 \%$ & 115 & $82,1 \%$ \\
Presentación de caso & 25 & $17,9 \%$ & 115 & $82,1 \%$ \\
Motivo de consulta & 32 & $22,9 \%$ & 108 & $77,1 \%$ \\
Diagnostico & 35 & $25,0 \%$ & 105 & $75,0 \%$ \\
Número de historia clínica & 41 & $29,3 \%$ & 99 & $70,7 \%$ \\
Cuestionario de salud & 118 & $84,3 \%$ & 22 & $15,7 \%$ \\
Examen estomatológico & 120 & $85,7 \%$ & 20 & $14,3 \%$ \\
Fecha de ingreso & 120 & $85,7 \%$ & 20 & $14,3 \%$ \\
Odontograma & 135 & $96,4 \%$ & 5 & $3,6 \%$ \\
Asignación de operador & 137 & $97,9 \%$ & 3 & $2,1 \%$ \\
Filiación & 138 & $98,6 \%$ & 2 & $1,4 \%$ \\
Evolución del tratamiento & 138 & $98,6 \%$ & 2 & $1,4 \%$ \\
\hline
\end{tabular}

Solo un aspecto (evolución del tratamiento) se encuentra registrada en el 100\% de historias clínicas evaluadas. 


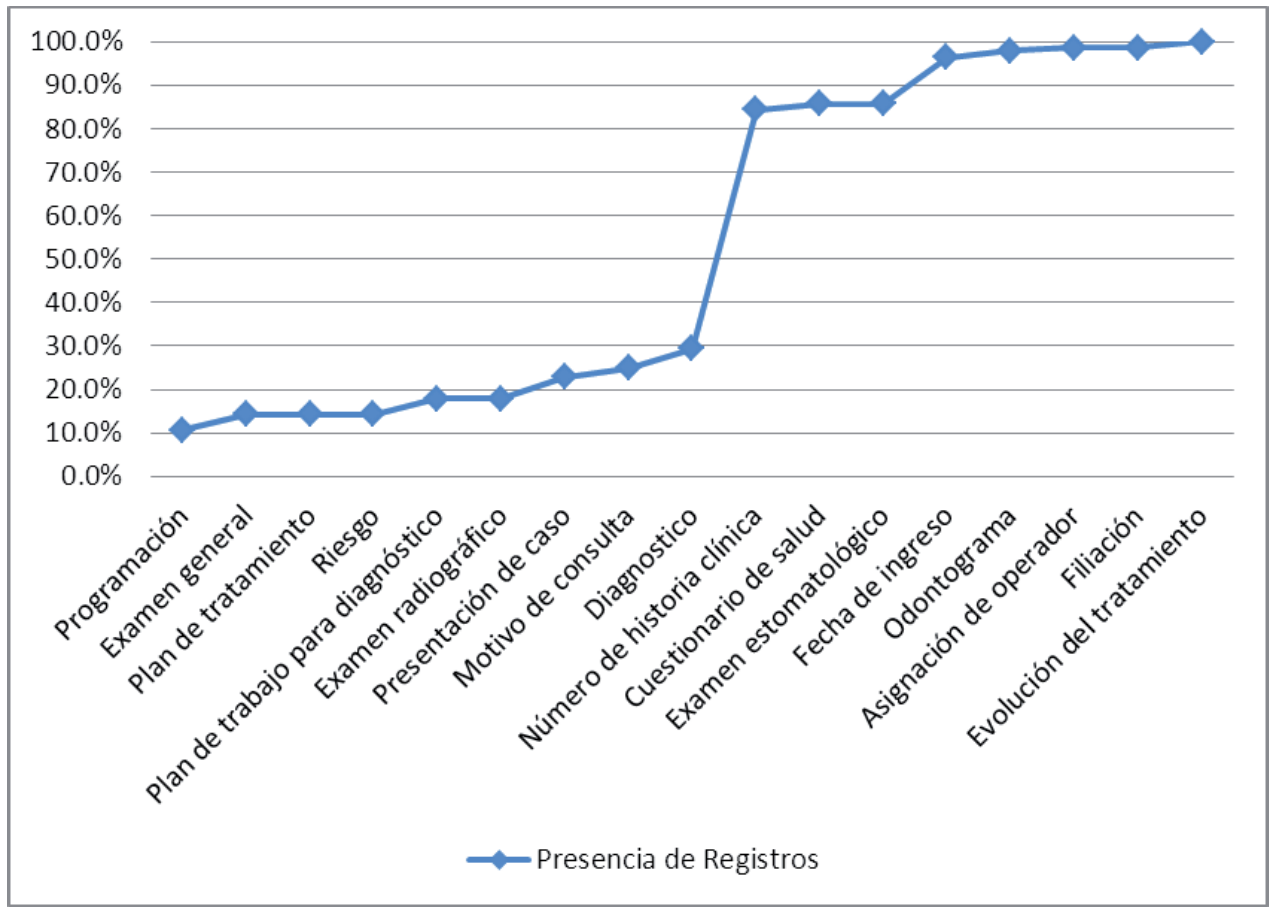

Gráfico 2. Distribución de registros presentes y completos en historias clínicas auditadas. Hay una relación inversamente proporcional entre la presencia y ausencia de registros en las historias clínicas.

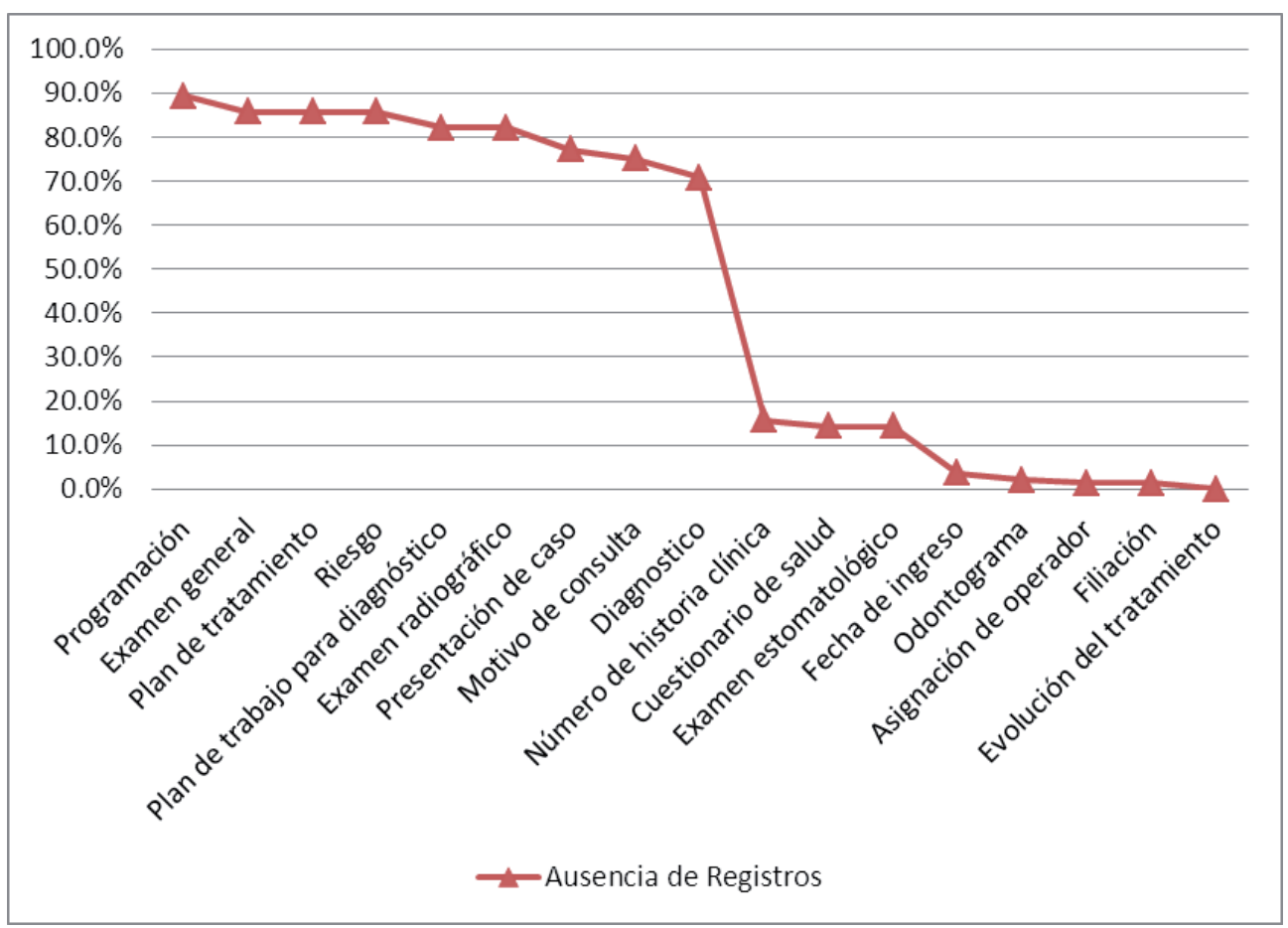

Gráfico 3. Distribución de registros ausentes e incompletos en historias clínicas auditadas.

Amplia brecha entre los registros con altos porcentajes de llenado completo Vs los registros con bajos porcentajes de llenado incompleto. 
Se registran algunos estudios sobre auditoria de historias clínicas, uno de ellos publicado en el 2006, en el cual se evalúa la calidad de los datos registrados en historias clínicas de atención ambulatoria de pacientes nuevos atendidos en consultorios externo de Pediatría General en el Hospital Nacional Cayetano Heredia $(\mathrm{HNCH})$, el estudio incluyó 384 historias clínicas y consideró: datos del paciente, anamnesis, examen físico, diagnóstico, plan de trabajo, tratamiento e identificación del médico tratante, realizando una medición dicotómica de la variable (presencia o ausencia de la variable), teniendo como resultados que el motivo de consulta, el peso y talla del paciente estuvieron presentes en todas las historias clínicas. La fecha, edad, y examen físico estuvieron presentes en más del $90 \%$, el nombre y número de historia clínica en menos del 50\%, mientras que las funciones biológicas y funciones vitales en menos del $20 \%$. El diagnóstico fue registrado en $99,2 \%$ y el plan de trabajo en $84,6 \%$. El tratamiento farmacológico se encontró completo en 7,4\%. La identificación del médico se encontraba completa sólo en $49,7 \%$. Con todo esto se concluyó que es necesario implementar mecanismos que permitan de manera efectiva a los médicos y equipos multidisciplinarios de atención en salud monitorizar y mejorar la calidad de la atención que brindan a sus pacientes (13).

Otra publicación al respecto en el mismo año 2006, evalúa el registro de historias clínicas de consulta externa, permitiendo identificar los errores o vacíos de información. El estudio incluye muestras aleatorias representativas de pacientes nuevos atendidos en cuatro hospitales públicos peruanos del Ministerio de Salud de la costa norte (Lambayeque), costa sur (Ica), sierra (Junín) y selva (Amazonas) (14).

En el campo odontológico, se tiene una revisión de expedientes dentales en Odontología Integrada de Adultos de la Facultad de Odontología de Sevilla (España) en el año 2004, con el propósito de averiguar si la documentación era adecuada, se consensuaron 46 criterios de calidad explícitos, en la fase de evaluación, se alcanzó el estándar de cumplimiento adecuado en 12 criterios. Como medida correctiva se elaboró un nuevo formato de historia clínica, que se utilizó durante 2 años. Luego de este período, se realizó la $2^{\mathrm{a}}$ fase de reevaluación, o auditoría post corrección, en la que se observó una importante mejoría en el número de criterios alcanzando el estándar de adecuado de 29 criterios (15).
En la literatura extranjera entre los años 1972 y 1979 se reportan muchos estudios de sistemas de tasación y garantía de calidad que han usado la historia clínica odontológica como fuente de datos (16). Destacan también estudios que desarrollaron sistemas de garantía de calidad confiando claramente en las historias clínicas odontológicas de los pacientes para evaluar la conveniencia y calidad de la asistencia suministrada (17).

Asimismo, en nuestro país, hace tres décadas se realizó un estudio de auditoria odontoestomatológica en los servicios del MINSA que marca un hito en la literatura latinoamericana, y en el cual adelantándose a la época, planteó una metodología similar a la de la auditoria médica, incorporando formatos de evaluación y escalas cuantitativas de valoración. Evaluó tres aspectos bastante importantes en los servicios odontológicos: La estructura (equipos), los procesos (directa a individuos) y los resultados (tiempo, complicaciones, costos) (18).

La historia clínica es uno de los elementos fundamentales para el trabajo médico y odontológico, y es la pieza clave en toda auditoría de servicios de salud. La experiencia ha demostrado un gran número de problemas en el manejo de las historias clínicas: ausencia de uniformidad, pérdida de historias, duplicidad, historias incompletas, radiografías extraviadas o mal conservadas, historias no actualizadas, anotaciones ilegibles, no presencia de un plan de tratamiento escrito, ausencia de firmas del paciente o del proveedor del servicio, firmas sin identificar, discrepancia entre los diagnósticosiniciales y los tratamientos realizados, falta de consentimiento por parte de los pacientes, tratamientos interrumpidos por causa desconocida, etc (19).

Todos estos problemas, errores u omisiones de información no registrada, parcialmente registrada o registrada de forma errónea, pueden repercutir -de forma directa o indirecta- en la duración del tratamiento, en su pertinencia u oportunidad y hasta generar eventos adversos debido a mala praxis, en otras palabras, afectar la calidad del servicio.

Los problemas en las historias clínicas también pueden perjudicar la actividad científica puesto que podemos obtener conclusiones erróneas si utilizamos los expedientes como fuentes de datos en nuestras investigaciones (20). Es por todo ello 
que las historias clínicas constituyen documentos de alto valor médico, gerencial, legal, académico y científico, como tal, la información en las mismas contribuye directa e indirectamente en la calidad de atención a los pacientes, optimiza la gestión de los establecimientos, protege los intereses legales del paciente, del personal de salud y del establecimiento y favorece la investigación y docencia universitaria.

\section{CORRESPONDENCIA:}

Jorge A. Manrique.

Calle Arquitecto Juan Benítez No 289 Departamento $\mathrm{N}^{\mathrm{o}} 301$.

Torres de Limatambo. San Borja. Lima. Perú.

Correo electrónico: jorgemanguz@hotmail.com

\section{REFERENCIAS BIBLIOGRÁFICAS}

1. Feld S, Rizzi CH, Goberna A. El control de la atención médica. Buenos Aires: López Libreros Editores; 1978

2. Osorio G, Sayes N, Fernández M. Auditoria médica: herramienta de gestión moderna subvalorada. Rev. Méd. Chile, 2002;130(2):226-9.

3. Frostick S, Ratford P, Wallace A. Introduction: En: Medical audit rationale and practicalities. Cambridge: Cambridge University; 1993.

4. Fraser R, Baker R, Lakhani M. Evidence-based clinical audit: an overview. En: Baker R, Fraser R, Lakhani M. Evidence-based audit in general practice. Oxford: Butterworth-Heinemann; 1998.

5. Ortega-Benito J. La auditoría médico-clínica (II). Definiciones y tipología. Barcelona: Medicina Clínica; 1994.

6. Jones $\mathrm{T}$, Cawthorn $\mathrm{S}$. What is clinical audit? Newmarket CB8 7LG, Reino Unido: Hayward Medical Communications; 2002.

7. Mata J, Martos E, Anzaldi M. Auditoría odontológica. Buenos Aires: Ediciones Dunken; 1996.

8. Garaycochea V. Auditoría médica. Pediátrica. 2000; $3(1): 27-28$.

9. Manrique J. Auditoría odontológica como herramienta para la garantía. Lima: Congreso Internacional - Master Challenge, Facultad de Estomatología, Universidad Peruana Cayetano Heredia; 2009.
10. Feinstein AR. Quality of data in the medical record. Computers and Biomedical Research. 1970; 3:426-435

11. Ruiz M, Benitez B. Tipología documental en las historias clínicas. Revista de Estudios Extremeños. 2003; 59(1):481-94.

12. Heredia C. Historia clínica pediátrica: Manual de procedimientos para el registro de datos. Lima, Perú. Facultad de Estomatología de la Universidad Peruana Cayetano Heredia, 2001.

13. Perat M, Llanos L, Cabello E, et al. Auditoría médica en la consulta externa pediátrica en un hospital general, Lima-Perú. Rev Med Hered. 2006;17(1):35-41.

14. Llanos L, Mayca J, Navarro G. Auditoría médica de historias clínicas en consulta externa de cuatro hospitales públicos peruanos. Rev Med Hered. 2006;17(4):220-6.

15. Martín M. Control de calidad en odontología integrada de adultos: Auditoría de historias clínicas. Sevilla: Fondos digitalizados de la Universidad de Sevilla; 2004

16. Friedman J, Schoen M. Audit of quality of dental care, a pilot study. J Public Health Dent. 1972; 32(4):214-24.

17. Marcus M, Koch L, Gersshen J. A record review model for assessing dental practices. CDA J. 1979; 7(10):51-4.

18. Duthie B. Audit: historical and future perspectives. En: Frostick SP, Radford PJ, Angus W. Medical audit rationale and practicalities. Cambridge: Cambridge University Press; 1993

19. Harrison A. Principles of internal medicine. 18ava. Ed. Mexico DF: Mc Graw Hill; 2012.

20. Lishner A. Auditoria odontoestomatológica en los Servicios del Ministerio de Salud. Tesis para optar el grado de Doctor en Odontologia. Lima, Perú. Universidad Nacional Mayor de San Marcos, 1977.

Recibido : 23/07/2013

Aceptado: 16/09/2013 